\title{
Oral immunization against hepatitis $B$ using bile salt stabilized vesicles (bilosomes)
}

\author{
Anshuman Shukla, Kapil Khatri, Prem N. Gupta, Amit K. Goyal, Abhinav Mehta and Suresh P. Vyas \\ Drug Delivery Research Laboratory, Department of Pharmaceutical Sciences, India.
}

Received October 12, 2007; Revised January 16, 2008; Accepted February 14, 2008; Published February 20, 2008;

\begin{abstract}
Purpose. Most of the presently available vaccines including hepatitis $B$ vaccines administered through parenteral route, fail to induce a mucosal antibody response. Therefore, oral immunization appears to be an effective and attractive alternative to parenteral immunization. However, the problem of degradation of antigen in the harsh and hostile environment of the gastrointestinal tract consequently requires larger doses and more frequent dosing of antigen. Furthermore, much larger doses can induce antigen tolerance. Therefore the purpose of the present study was firstly to overcome these problems by the use of bile salt stabilized vesicles (bilosomes) and HBsAg as the model antigen, which could provide both protection to the antigen as well as enable transmucosal uptake and subsequent immunization. Another purpose of this study was to determine the dose that could produce serum antibody titres against hepatitis $B$ via the oral route compared to those following intramuscular immunization. Methods. In the present study bilosomes containing recombinant hepatitis B surface antigen were prepared by a lipid cast film method. HBsAg loaded bilosomes were characterized in vitro for their shape, size, percent antigen entrapment and stability. Fluorescence microscopy was carried out to confirm the uptake of bilosomes by gut associated lymphoid tissues (GALT). The in vivo part of the study comprised estimation of anti-HBsAg IgG response in serum and anti-HBsAg sIgA in various body secretions using specific ELISA techniques following oral immunization with low dose loaded bilosomes (B1, $10 \mu \mathrm{g}$ ), intermediate dose loaded bilosomes (B2, $20 \mu \mathrm{g}$ ) and high dose loaded bilosomes (B3, $50 \mu \mathrm{g}$ ) in BALB/c mice. Results. Fluorescence microscopy suggested that there was an increase in fluorescence intensity following the uptake of bilosomes entrapped FITC-BSA in gut associated lymphoid tissues. The high dose HBsAg bilosomes (B3, $50 \mu \mathrm{g}$ ) produced comparable anti-HBsAg IgG levels in serum to those observed in the case of
\end{abstract}

intramuscular administration of alum adsorbed HBsAg (10 $\mu g)$. In addition, the bilosomal preparations elicited measurable sIgA in mucosal secretions, where the highest responses were observed with high dose HBsAg bilosomes (B3, $50 \mu \mathrm{g})$ and as expected, intramuscular administered alum adsorbed HBsAg $(10 \mu \mathrm{g})$ failed to elicit such responses. Conclusions. HBsAg loaded bilosomes produced both systemic as well as mucosal antibody responses upon oral administration. Furthermore, bilosomes with a five times higher dose upon oral administration produced comparable serum antibody titres to those obtained after intramuscular immunization without the induction of systemic tolerance.

\section{INTRODUCTION}

Viral hepatitis has become a global public health problem. Hepatitis B virus (HBV) causes both acute and chronic infections. The wide clinical spectrum of HBV infection ranges from sub clinical to acute symptomatic hepatitis; from an inactive hepatitis B surface antigen (HBsAg) carrier state to liver cirrhosis and its complications during the chronic phase (1). About $90 \%$ of infants who acquire HBV infection from their mothers at birth become chronically infected. More than 3,000 persons reportedly die of Hepatitis B-related cirrhosis each year in the United States (2). Persons with chronic HBV infection are at 12 to 300 times higher risk of hepatocellular carcinoma than non-carrier individuals (2).

HBV is transmitted on parenteral or mucosal exposure to HBsAg positive body fluids generally from HBV infected persons (3).

Corresponding Author: Prof. S. P. Vyas, Drug Delivery Research Laboratory, Department of Pharmaceutical Sciences, E-mail: vyas_sp@rediffmail.com 
Currently, the available hepatitis B vaccine is administered parenterally and induces only systemic immune response. The antibodies produced as systemic immune response are unable to provide protection at the level of mucosa, which is the major entry site for most infectious agents including HBV.

Mucosal immunization via the oral route utilizing appropriate delivery systems can be an effective and attractive alternative to this conventional parenteral immunization. Oral administration of antigens induces production of sIgA, not only at the site of antigen application, but also in other external secretions due to the dissemination of antigen-sensitized cells to other tissues (4). Oral immunization utilizing delivery systems can cause induction of both systemic as well as mucosal immune responses.

Apart from this, oral immunization also offers a number of inherent advantages over the parenteral route that include ease and convenience of administration, increased patient compliance, and minimal side effects and thus a greater possibility of frequent boosting.

The problem associated with oral delivery of antigen, i.e. the denaturation and degradation in the hostile environment of gastrointestinal tract, consequently requires larger and more frequent dosing of antigen which further leads to oral tolerance (4); this can be overcome by utilizing appropriate delivery systems (5-11).

Previously, oral administration of antigens using bilosomes have showed effective stimulation of both systemic and mucosal immune responses suggesting bilosomes to be a promising and potential carrier system for oral immunization (11-14).

The major objective of the present study was to determine the dose that could produce serum antibody titres against hepatitis B via the oral route using bilosomes which were comparable to those following intramuscular immunization. In this study recombinant hepatitis B surface antigen containing bilosomes with different entrapped doses were prepared and assessed for their potential to induce of both mucosal and systemic immune responses.

\section{MATERIALS AND METHODS}

\section{Materials}

Sorbitan tristearate and cholesterol were purchased from Fluka Chemika, India. Dicetyl phosphate, sodium deoxycholate, FITC-BSA and sephadex G-100 were procured from Sigma Chemical Co. (St. Louis, MO, USA). Recombinant hepatitis B surface antigen was obtained from Shantha Biotechnics Ltd. (Hyderabad, India) as a gift sample. AUSZYME monoclonal kit was obtained from Abbott Laboratories, USA. All other chemicals and reagents were of analytical grade and purchased from local suppliers unless otherwise mentioned.

\section{Preparation of Hepatitis B Surface antigen (HBsAg) loaded bilosomes}

Bile salt stabilized vesicles (bilosomes) were prepared as previously described by Conacher et al. (11) with some modifications. Briefly sorbitan tristearate, cholesterol and dicetyl phosphate in 7:3:1 molar ratios were dissolved in $10 \mathrm{ml}$ chloroform in a round-bottomed flask. The chloroform was removed under reduced pressure using a rotatory evaporator to produce a thin film on the side of flask. The film was subsequently hydrated with $3.5 \mathrm{ml}$ phosphate buffer saline (PBS, pH 7.4), containing $100 \mathrm{mg}$ of sodium deoxycholate along with $400 \mu \mathrm{g}$ (low dose, 10 $\mu \mathrm{g} / \mathrm{dose}, \mathrm{B} 1$ ), $800 \mu \mathrm{g}$ (intermediate dose, 20 $\mu \mathrm{g} /$ dose, B2) and $2000 \mu \mathrm{g}$ (high dose, $50 \mu \mathrm{g} /$ dose, B3) of hepatitis B surface antigen (HBsAg), to produce HBsAg loaded bilosomes. The total preparation volume was than made up to $4 \mathrm{ml}$ with PBS. The bilosomes were then sized by extrusion through $200 \mathrm{~nm}$ pore membrane (Millipore, USA). The unentrapped antigen and sodium deoxycholate were removed by mini column centrifugation method using sephadex G-100 column as described by Fry et al. (11). Fractions were collected and challenged with Triton X-100 (0.2\% v/v). The samples were diluted in order to fall within the standard curve $(10 \mu \mathrm{g} / \mathrm{ml}-100 \mu \mathrm{g} / \mathrm{ml})$. HBsAg was measured using micro BCA protein estimation kit (Genei, India). Experiment was repeated three times using a fresh column each time.

\section{Characterization of bilosomes}

The morphological examination of the bilosomes was performed using a transmission electron microscope (Philips CM-10, Netherlands) after negative staining of the samples with phosphotungustic acid solution $(2 \% \mathrm{w} / \mathrm{v})$. The mean particle size was determined by a photon correlation spectroscopy using a Malvern 
Zetasizer Nano ZS 90 (Malvern Instruments Co., U.K.).

\section{Stability in simulated gastric fluid, simulated intestinal fluid and bile salt solutions}

Stability of HBsAg loaded bilosomes was determined in simulated gastric fluid (SGF, $\mathrm{pH}$ 1.2), simulated intestinal fluid (SIF, $\mathrm{pH} 7.5$ ) and different concentrations of bile salt (5 and 20 $\mathrm{mM}$ ) by addition of $1.8 \mathrm{ml}$ of different solutions to $0.2 \mathrm{ml}$ of bilosomal formulation. After $2 \mathrm{~h}$ samples were withdrawn and unentrapped $\mathrm{HBsAg}$ was removed by minicolumn centrifugation method using sephadex G-100 column as described by Fry et al. (15). Unentrapped HBsAg free bilosomes were challenged with Triton X$100(0.2 \% \mathrm{v} / \mathrm{v})$ and HBsAg was estimated using AUSZYME $^{\circledR}$ monoclonal kit (Abbott Laboratories, USA).

\section{Fluorescence microscopy}

Fluorescence microscopy was performed for the confirmation of deposition of bilosomes in the GALT. FITC-BSA was used as a fluorescent marker and was loaded into the bilosomes. After 5 $\mathrm{h}$ (4) of oral administration of bilosomal formulation, the animals were sacrificed, the small intestine was removed, and cut and microtomy was performed. The sections of around $3 \mu \mathrm{m}$ thickness were viewed under a fluorescence microscope (Nikon Eclipse E 600) $(n=3)$. Control animals were given the equivalent amount of unentrapped FITC-BSA (5mg in 500 $\mu \mathrm{l}$ PBS (pH 7.4)) orally and microtomy was performed.

\section{Immunization and sample collection}

Female BALB/c mice of 6-8 weeks age, weighing $15-20 \mathrm{~g}$ were used for in vivo studies. Animals were housed in groups of five with free access to food and water. They were deprived of any food intake for $3 \mathrm{~h}$ prior to immunization. The study protocols followed as approved by Institutional Animals Ethical Committee of Dr. Hari Singh Gour University, Sagar. The studies were carried out according to the guidelines of the Council for the Purpose of Control and Supervision of Experiments on Animals, Ministry of Social Justice and Empowerment, Government of India. The mice were immunized by intragastric lavage, following the protocol: three primary inoculations for three consecutive days and boosting after 3 weeks was done. In order to administer $10 \mu \mathrm{g}$

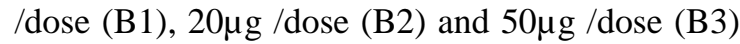
the film was hydrated with $3.5 \mathrm{ml}$ PBS containing $400 \mu \mathrm{g}$ of HBsAg (group B1), $800 \mu$ g of HBsAg (group B2), $2000 \mu \mathrm{g}$ of HBsAg and then final volume was made up to $4.0 \mathrm{ml}$, that is $100 \mu \mathrm{g}$ of HBsAg was present in $1 \mathrm{ml}$ (group B1), 200 $\mathrm{gg}$ of HBsAg was present in 1ml (group B2), 500 $\mu$ g of HBsAg was present in $1 \mathrm{ml}$ (group B3) respectively. Since the entrapment was found to be around $20 \%$ this means $20 \mu \mathrm{g} \mathrm{HBsAg} / \mathrm{ml}, 40 \mu \mathrm{g}$ $\mathrm{HBsAg} / \mathrm{ml}, 100 \mu \mathrm{g} \mathrm{HBsAg} / \mathrm{ml}$ was entrapped in bilosomes of Group B1, Group B2 and Group B3 respectively. Moreover, $0.5 \mathrm{ml}$ bilosomes was orally administered, thus $10 \mu \mathrm{g} /$ dose (B1), $20 \mu \mathrm{g}$

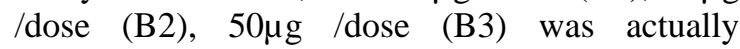
administered.

The mice were immunized by intragastric administration of formulations with $0.5 \mathrm{ml}$ low dose HBsAg bilosomes (B1, 10 $\mu$ g/dose, group 1); $0.5 \mathrm{ml}$ intermediate dose HBsAg bilosomes (B2, $20 \mu \mathrm{g} /$ dose, group 2); and $0.5 \mathrm{ml}$ high dose HBsAg bilosomes (B3, $50 \mu \mathrm{g} /$ dose, group 3) for three consecutive days. Booster immunization was done after 3 weeks. The control group (group 4) received a $10 \mu \mathrm{g}$ dose of alum-adsorbed $\mathrm{HBsAg}$ intramuscularly on day 0 and a booster 3 weeks after primary immunization.

Samples of serum and secretions (saliva, vaginal fluid and intestinal lavage) were collected from immunized animals on day 0 before immunization. Blood was collected by retroorbital plexus under light ether anesthesia after 14, 28, 42 and 56 days of booster dosing and sera was stored at $-40^{\circ} \mathrm{C}$ until tested by ELISA for antibody titres. The intestinal lavage, vaginal and salivary secretions were collected after 5 weeks of booster immunization. For collection of saliva, mice were administered $0.2 \mathrm{ml}$ sterile solution of pilocarpine (10 mg/ml) intraperitoneolly (IP), and 20 min later, the saliva was collected using capillary tube. Intestinal lavage was collected using the technique reported by Elson et al. (16). Briefly, four doses of $0.5 \mathrm{ml}$ lavage solution ( $\mathrm{NaCl} 25 \mathrm{mM}, \mathrm{Na}_{2} \mathrm{SO}_{4} 40 \mathrm{mM}, \mathrm{KCl} 10 \mathrm{mM}$, $\mathrm{NaHCO}_{3} 20 \mathrm{mM}$ and polyethylene glycol MW 3350; $48.5 \mathrm{mM}$ ) were administered intragastrically at 15 min. intervals using a blunt tipped feeding needle. Thirty minutes after the last dose the mice were given $0.2 \mathrm{ml}$ pilocarpine $(10 \mathrm{mg} / \mathrm{ml})$ IP. A discharge of intestinal contents was carefully collected for the next 20 minutes. Vaginal secretions were collected by using a pipettor to douche the mice with $0.1 \mathrm{ml}$ of PBS (pH 7.4), which was then aspirated back into the pipette tip 
and used for the determination of antibody levels. These fluids were stored with $100 \mathrm{mM}$ phenylmethyl sulfonyl fluoride (PMSF) as a protease inhibitor at $-40^{\circ} \mathrm{C}$ until tested by ELISA for secretory antibody (sIgA) levels.

\section{Measurement of specific IgG and IgA responses}

Antibody responses in immunized animals were monitored using a microplate ELISA. Microtiter plates (Nunc-Immuno Plate ${ }^{\circledR}$ Fb 96 Mexisorp, NUNC) were coated with $100 \mu \mathrm{l} /$ well of $10 \mu \mathrm{g} / \mathrm{ml}$ HBsAg in PBS (pH 7.4) and incubated overnight at $4^{\circ} \mathrm{C}$. The plates were washed three times with PBS-Tween $20(0.05 \%, \mathrm{v} / \mathrm{v})(\mathrm{PBST})$ and blocked with PBS-BSA $(3 \% \mathrm{w} / \mathrm{v})$ for $2 \mathrm{~h}$ at $37^{\circ} \mathrm{C}$, followed by washing with PBS-T. The serum/ body fluids were serially diluted with PBS and $100 \mu \mathrm{l}$ of each sample was added to each well of coated ELISA plates. The plates were incubated for $1 \mathrm{~h}$ at room temperature and washed three times with PBST. One hundred microliters of peroxidase labeled goat anti-mouse IgG/ IgA (1:1,000 dilution, Sigma, USA) was added to each well. The plates were covered and after incubation for $1 \mathrm{~h}$ at room temperature washing was repeated. One hundred microliters of tetramethyl benzidine (TMB- $\mathrm{H}_{2} \mathrm{O}_{2}$ ) solution was added to each well followed by addition of $50 \mu \mathrm{l}$ of $\mathrm{H}_{2} \mathrm{SO}_{4}$ after 90 min. After 15 min incubation, the plate was read at $450 \mathrm{~nm}$ using a plate reader (Biorad, USA). End point titers were expressed as the log of the reciprocal of the last dilution, which gave an optical density (OD) at $450 \mathrm{~nm}$ above the OD of negative controls.

\section{Statistical analysis}

Analysis of antibody titers was performed on logarithmically transformed data and the data were presented along with standard deviations (S.D.). Student's t-test was used to compare mean values of different groups. Multiple comparisons were made using one way analysis of variance (ANOVA) followed by post hoc analysis using Dunnet test. Statistical significance was considered at $\mathrm{P}<0.05$.

\section{RESULTS}

\section{Characterization of bilosomes}

The TEM photomicrographs (Figure I) clearly indicated that vesicles were unilamellar and spherical in shape. The mean particle size as determined by photon correlation spectroscopy using a Malvern Zetasizer, Nano ZS 90 (Malvern Instruments Co., U.K.) was found to be $204 \pm 18$ $\mathrm{nm}$. The amount of HBsAg entrapped in the bilosomes was found to be around $18-22 \%$ of the amount added.

\section{Stability studies}

Stability of the formulations was assessed in simulated gastric fluid (SGF, $\mathrm{pH}$ 1.2) and simulated intestinal fluid (SIF, $\mathrm{pH}$ 7.5). It was found that in simulated gastric fluid (SGF, pH 1.2) around $90 \%$ of HBsAg was retained in the vesicles and in simulated intestinal fluid (SIF, $\mathrm{pH}$ 7.5) around 95\% HBsAg was retained in the vesicles.

The formulations were also tested in $5 \mathrm{mM}$ and $20 \mathrm{mM}$ bile salt solutions. It was found that around $95 \%$ of HBsAg was retained in the vesicles at $5 \mathrm{mM}$ concentration and $85 \%$ of HBsAg was retained in the vesicles at $20 \mathrm{mM}$ concentration.

\section{Fluorescence microscopy}

Fluorescence microscopy revealed that after administration of FITC-BSA loaded bilosomes the localized fluoresce in the GALT region was much higher (Figure II B) compared to sections in which unentrapped FITC-BSA was administered orally (Figure IIA). This indicated the effective uptake of the bilosomes by the GALT (M Cells) especially to and through Peyer's patches.

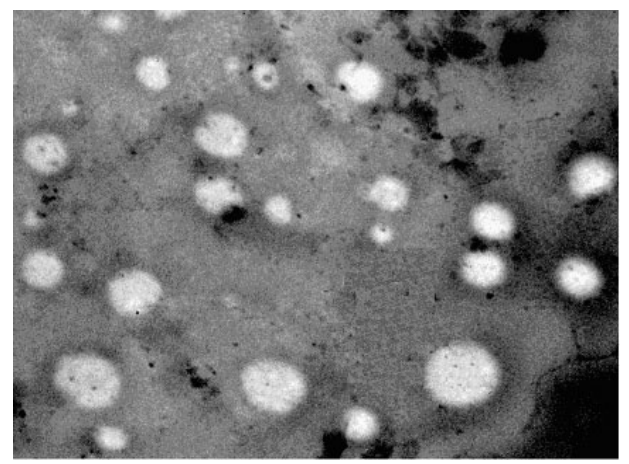

Figure 1. Photomicrograph of TEM of bilosomes. 

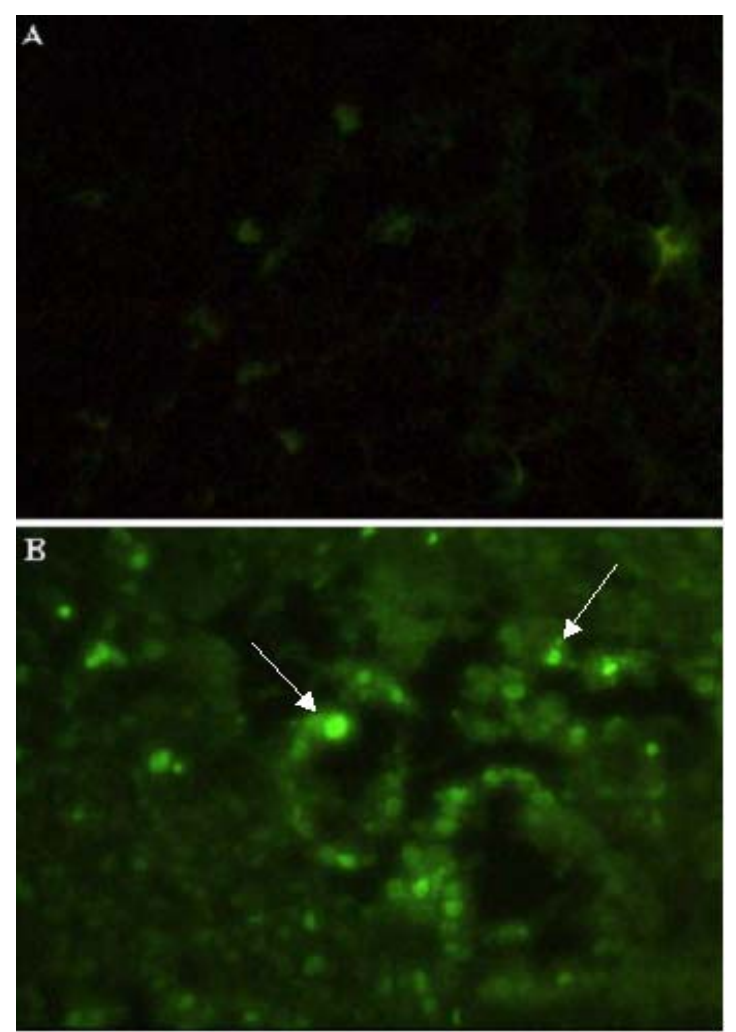

Figure II: Fluorescent image of small intestine (GALT) after the uptake of

(A) Unentrapped FITC-BSA administered orally

(B) FITC-BSA entrapped in bilosomes administered orally.

Arrow showing uptake of bilosomes by Peyer's Patches.

\section{Immunological studies}

The serum anti-HBsAg titre obtained after oral administration of B3 (i.e. high dose, 50 $\mu \mathrm{g} /$ dose) was comparable with titres obtained after intramuscular administration of alum adsorbed HBsAg (10 $\mu$ g, control group) but the responses obtained following oral administration of B3 (i.e. high dose, 50 $\mu \mathrm{g} /$ dose $)$ were significant $(\mathrm{p}<0.01)$ when compared with B1 (i.e. low dose, $10 \mu \mathrm{g} /$ dose). The systemic immune responses are graphically presented in Figure III.

All the orally administered formulations produced significant sIgA responses $(p<0.05)$ in mucosal secretions when compared with alum adsorbed HBsAg (control group), which was administered intramuscularly. Alum adsorbed formulations did not elicit detectable sIgA in mucosal secretions. The mucosal immune response measured as IgA titres is graphically shown in Figure IV.

\section{DISCUSSION}

In this study, transmission electron microscopy demonstrated that the vesicles were unilamellar and spherical in shape. The amount of antigen entrapped in the bilosomes was comparable with the data for other vesicular formulation such as liposomes incorporating HBsAg (17).

In the case of oral delivery of vaccines, the antigen is exposed to the acidic environment in the stomach and degrading enzymes in the intestinal tract. Therefore the stability determination of the formulations in simulated gastric fluid (pH 1.2), simulated intestinal fluid (pH 7.5) and in different bile salt concentration was carried out in order to assess the ability of the bilosomes to withstand various bioenvironmental stresses as well as to retain the stability of antigen. The stability was assessed in the terms of percentage antigen retained in the vesicles and the studies demonstrated significant stability in simulated fluids as well as in different bile salt concentrations.

Fluorescence microscopy demonstrated effective and efficient uptake of bilosomes by the GALT and therefore it can be concluded that bilosomes are efficient in transporting vaccines to the Peyer's patches, resulting in both mucosal and systemic immune responses. Oral tolerance induction is a key feature of intestinal immunity, generating systemic nonresponsiveness to ingested antigens (18). Oral tolerance may also be dependent on the frequency of antigen administration and the dose (4). However, a fine balance is necessary between the mucosal delivery of antigens and induction of tolerance (19). This study demonstrated effective and significant stimulation of both systemic and mucosal immune responses after oral administration of HBsAg loaded bilosomes and thus the goal of obviating tolerance was achieved successfully. Furthermore, bilosomes with higher dose of HBsAg (B3, $50 \mu \mathrm{g} /$ dose, oral) produced comparable anti-HBsAg IgG antibody titre responses compared with responses to intramuscular injection of $10 \mu \mathrm{g}$ of alum adsorbed HBsAg (control group) in addition to a mucosal antibody response.

Production of HBsAg-specific mucosal IgA antibodies is important for protection from mucosally transferred hepatitis B virus (20) and this study successfully demonstrated that all the bilosomal preparations elicited mucosal immune responses, with the highest response elicited 


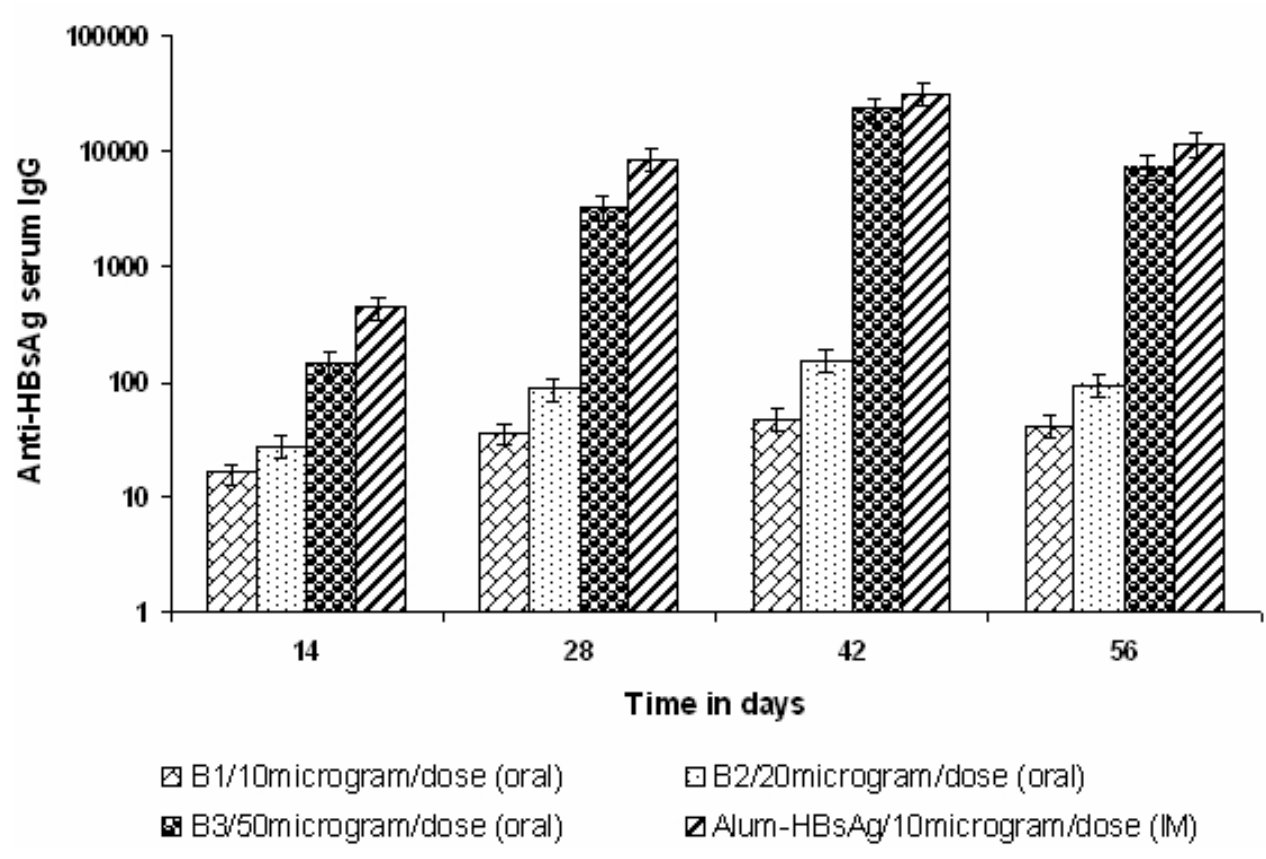

Figure III: Serum anti-HBsAg IgG profile of mice immunized orally with different formulations. The serum was collected after 14, 28, 42 and 56 days of boosting. Values are expressed as mean \pm S.D. ( $n=5$ ). Multiple comparisons were made using one way analysis of variance (ANOVA) followed by post hoc analysis using Dunnet test. Statistical significance was considered at $\mathrm{p}<0.05$.
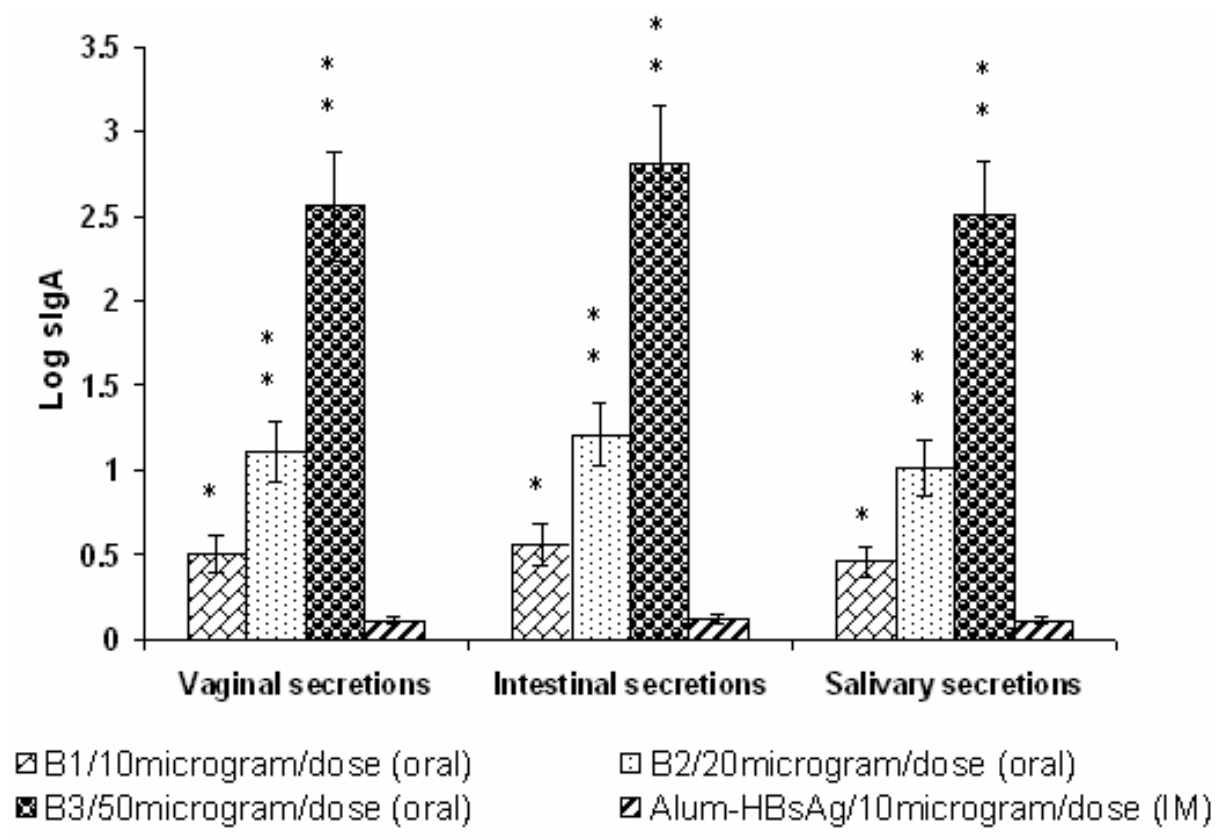

Figure IV: Secretory IgA level in vaginal, intestinal and salivary secretions of mice immunized orally with different formulations after 5 weeks of boosting. Values are expressed as mean \pm S.D. $(n=5)$. Multiple comparisons were made using one way analysis of variance (ANOVA) followed by post hoc analysis using Dunnet test. Alum HBsAg vs B1, $(\mathrm{p}<0.05 *)$, Alum HBsAg vs B2, $(\mathrm{p}<0.01 * *)$, Alum HBsAg vs B3, $(\mathrm{p}<0.01 * *)$. 
in the case of bilosomes with a higher dose of HBsAg (B3, $50 \mu \mathrm{g} /$ dose, oral). The intramuscular injection of $10 \mu \mathrm{g}$ of alum absorbed HBsAg (control group) was unable to produce detectable mucosal sIgA antibody response, which is not unexpected.

In conclusion, the bile salt stabilized vesicles (bilosomes) are a promising carrier for oral immunization of HBsAg and five times higher entrapped dose is required using bilosomes to produce a comparable systemic antibody response with an additional benefit of secretory mucosal protection. Thus, HBsAg loaded bilosomes can provide a needle free, painless approach for immunization against hepatitis B, thereby increasing patient compliance and consequently increasing vaccination coverage.

\section{ACKNOWLEDGEMENTS}

The authors are grateful to Shantha Biotechnics Ltd., Hyderabad for providing the gift sample of recombinant hepatitis B surface antigen (HBsAg). The authors are thankful to AIIMS, New Delhi for carrying out the transmission electron microscopy of the formulations. Author AS is thankful to AICTE for providing financial assistance for a Junior Research Fellowship.

\section{REFERENCES}

[1]. Fattovich G. Natural history of hepatitis B. J Hepatol, 39:S50-58, 2003.

[2]. CDC. A comprehensive immunization strategy to eliminate transmission of hepatitis $B$ virus infection in the United States: recommendations of the Advisory Committee on Immunization Practices (ACIP). Part 1: Immunization of infants, children and adolescents. MMWR, 46 (No.RR-18):1-42, 1997.

[3]. Hilleman MR. Critical overview and outlook: pathogenesis, prevention and treatment of hepatitis and hepatocarcinoma caused by hepatitis B virus. Vaccine, 21: 4626-4649, 2003.

[4]. Shalaby WSW. Development of oral vaccines to stimulate mucosal and systemic immunity: barriers and novel strategies. Clin Immunol Immunopath, 74(2):127-134, 1995.

[5]. Borges O, Borchard G, Verhoef JC, Sousa A, and Junginger HE. Preparation of coated nanoparticles for a new mucosal vaccine delivery system. Int J Pharm, 299:155-165, 2005.

[6]. Lubben IM, Verhoef JC, Aelst AC, Borchard $\mathrm{G}$, and Junginger HE. Chitosan microparticles for oral vaccination: preparation, characterization and preliminary in vivo uptake studies in murine peyer's patches.Biomaterials, 22:687-694, 2001.

[7]. Bowersock TL, Esch HH, Suckow M, Guimond P, Martin S, Borie D, Torregrosa S, Park $\mathrm{H}$, and Park K. Oral vaccination of animals with antigens encapsulated in alginate microspheres. Vaccine, 17:1804-1811, 1999.

[8]. Hori M, Onishi H, and Machida Y. Evaluation of Eudragit-coated chitosan microparticles as an oral immune delivery system. Int J Pharm, 297:223-234, 2005.

[9]. Lavellea EC, Sharif S, Thomasb NW, Holland $\mathrm{J}$ and Davis SS. The importance of gastrointestinal uptake of particles in the design of oral delivery systems. Advanced Drug Delivery Reviews, 18:5-22, 1995.

[10]. Singh M, and Hagan DO. The preparation and characterization of polymeric antigen delivery systems for oral administration. Advanced Drug Delivery Reviews, 34:285-304, 1998.

[11]. Conacher M, Alexander J, and Brewer JM. Oral immunisation with peptide and protein antigens by formulation in lipid vesicles incorporating bile salts (bilosomes). Vaccine 19:2965-2974, 2001.

[12]. Mann JFS, Ferro VA, Mullen AB, Tetley L, Mullen M, Carter KC, Alexander $\mathrm{J}$ and Stimson WH. Optimization of a lipid based oral delivery system containing $\mathrm{A} /$ Panama influenza haemagglutinin. Vaccine, 22:24252429, 2004.

[13]. Singh P, Prabakaran D, Jain S, Mishra V, Jaganathan KS, and Vyas SP. Cholera toxin B subunit conjugated bile salt stabilized vesicles (bilosomes) for oral immunization. Int $\mathrm{J}$ Pharm, 278:379-390, 2004.

[14]. Mann JFS, Scales HE, Shakir E, Alexander JA, Carter KC, Mullen AB, and Ferro VA. Oral delivery of tetanus toxoid using vesicles containing bile salts (bilosomes) induces significant systemic and mucosal immunity. Methods, 38:90-95, 2006.

[15]. Fry DW, White JC, and Goldman ID. Rapid separation of low molecular weight solutes from liposomes without dilution. Anal Biochem, 90:809-815, 1978.

[16]. Elson CO, Ealding W and Lefkowitz J. A lavage technique following repeated measurement of IgA antibody in mouse intestinal secretions. J Immunol Methods, 67:101-108, 1984.

[17]. Sanchez Y, Matiu II, Dreesman GR, Kramp W, Six HR, Hollinger B, and Melnick JL. Humoral and cellular immunity to hepatitis B virus-derived antigens: comparative activity of freund complete adjuvant, alum and liposomes. Infect Immun, 30:728-733, 1980. 
[18]. Worbs T, Bode U, Yan S, Hoffmann MW, Hintzen G, Bernhardt G, Forster R, and Pabst O. Oral tolerance originates in the intestinal immune system and relies on the antigen carriage by dendritic cells. J Exp Med, 203(3):519-527, 2006.

[19]. Holmgren J, and Czerkinsky C. Mucosal immunity and vaccines. Nat Med, 11(4 suppl):S45-S53, 2005.
[20]. Isaka M,Yasuda Y, Mizokami M,Kozuka S,Taniguchi T,Matano K,Maeyama J,Mizuno K,Morokuma K,Goto N,and Tochikubo K.Mucosal immunization against hepatitis B virus by intranasal co-administration of recombinant hepatitis $\mathrm{B}$ surface antigen and recombinant cholera toxin $\mathrm{B}$ subunit as an adjuvant.Vaccine,19:1460-1466,2001. 